\title{
Batch Measurement Extremum Seeking Control of Distributed Energy Resources to Account for Communication Delays and Information Loss
}

\author{
Michael D. Sankur \\ Lawrence Berkeley Lab \\ msankur@lbl.gov
}

\author{
Maxime Baudette \\ Lawrence Berkeley Lab \\ baudette@lbl.gov
}

\author{
Jason S. MacDonald \\ Lawrence Berkeley Lab \\ jsmacdonald@1bl.gov
}

\author{
Daniel B. Arnold \\ Lawrence Berkeley Lab \\ dbarnold@lbl.gov
}

\begin{abstract}
Distributed Energy Resources (DER) have great potential to enhance the operation of electric power distribution systems. Previously, we explored the use of 2 Dimensional Extremum Seeking (2D-ES) control algorithms to enable model-free optimal control of DER to provide grid services to both the distribution and transmissions systems. Motivated by preliminary deployments of DER managed by $2 D$-ES algorithms in hardware-in-the-loop tests and in operational distribution grids, in this work, we extend the control scheme to accommodate communication delays and information loss. We propose a modification to the 2D-ES scheme to allow for the processing of batches of possibly noncontiguous objective function measurements at unknown and possibly uneven intervals. We provide a proof of the convergence of the batch $2 D-E S(2 D-B E S)$ scheme when optimizing a generic convex objective function, as well as simulation results that demonstrate the suitability of the approach for substation active and reactive power target tracking.
\end{abstract}

\section{Introduction}

Optimal control of Distributed Energy Resources (DER) is a topic that has garnered great interest among researchers and engineers in the electric power community. Due to the presence of generation sources at the very fingertips of the distribution grid, both transmission and distribution system operators and planners are eager to incorporate DER to improve many aspects of the electric grid.

Within the research community, optimal control of

Michael D. Sankur, Maxime Baudette, Jason S. MacDonald, and Daniel B. Arnold are with Lawrence Berkeley National Laboratory. The work described herein was funded through the Office of Energy Efficiency \& Renewable Energy's Enabling Extreme Real-Time Grid Integration of Solar Energy (ENERGISE) Program in the U.S Department of Energy under Contract No. DE-AC02-05CH11231.
DER has been extensively researched and a variety of approaches have been put forth as a means to manage DER active/reactive power injections to provide a variety of grid services. The work of [1] proposes a distributed control framework to enable DER to track single-phase AC optimal power flow solutions using the Alternating Direction Method of Multipliers (ADMM). The authors of [2] consider a multi-timescale stochastic volt/var control method capable of controlling legacy voltage regulation systems as well as DER. Additional recent strategies for DER control are cataloged in [3].

A drawback of many approaches for optimally managing DER relies on the need of exogenous information, such as utility network topological data as well as global knowledge of real-time nodal power consumption, to facilitate the optimization. In the past, the authors of this paper have explored the use of Extremum Seeking Control [4] to manage DER. Extremum Seeking control (ESC) is an attractive alternative to many other real-time optimization strategies as the approach does not rely on explicit knowledge of the objective function and/or plant being optimized. Extremum Seeking utilizes periodic or stochastic perturbations of the parameters being optimized to seek the minimizer(s) of a mathematical program.

In the scheme, individual 2D-ES controllers, each managing an independent DER, modulate DER active and reactive power injections sinusoidally. The sinusoidal injections propagate throughout the system resulting in system voltages and power flows that also exhibit this oscillatory behavior. Measurements of system quantities (voltages and power flows) at locations where control action is desired are then collected by a centralized entity and used to compute a global objective function. The value of this objective function at each timestep is then broadcast to the individual 2D-ES controllers, who can then extract the gradient of their active/reactive power injection on the objective function. This gradient information is used by the controllers to update their active and reactive power 
setpoints at the next timestep. The proposed control scheme aggregates a selection of DER participating to optimize the same objective. While it is assumed that participating DER are contracted by the utility or aggregator to manage a common objective, it is not a requirement that all DER in the network participate in the 2D-ES scheme.

Our past work has focused on the development of a 2-Dimensional Extremum Seeking (2D-ES) approach to independently manage DER active and reactive power injections [5] to optimize a distribution feeder-level objectives, such as substation real power tracking and voltage management. Additionally, we have extended the approach to incorporate a decaying perturbation signal in the vicinity of the global objective function value [6]. The technical soundness of the approach has recently been validated by a group from Sandia National Lab, which implemented and tested the approach in power hardware-in-the-loop (PHIL) experiments [7].

The power hardware-in-the-loop tests highlighted several aspects of the 2D-ES approach that need to be addressed given the limitations of present-day hardware and communications systems, into which the 2D-ES algorithms are to be implemented. In particular, extremum seeking control's ability to converge rapidly on the objective's minima is dependent on the speed at which a resource can perturb the system through the probe signal. With the initial incarnation of extremum seeking the probe is limited by the speed at which an inverter can receive and respond to control commands, the speed that measurements can be collected to form the centralized objective, and the speed at which the objective signal can be broadcast to the ESC resources. As LBNL and partners at Sandia [7] have tested extremum seeking with inverters in multiple ongoing projects, these dependencies have manifested themselves repeatedly and forced the system to operate at much slower speeds than is achievable in simulation. In order to address these issues, the authors propose separating the functions of probing and setpoint generation to allow for the probe to be provided from an external source or command, breaking the interdependency between the communication speed restrictions and the probing frequency.

The work presented herein alters the formulation of ESC to mitigate the impacts of communication and response delays between the central entity constructing the objective, the distributed ES controllers, and their associated DER. By decoupling the probing process and setpoint generation in the control formulation, implementations of extremum seeking can reduce the frequency in which communications between these devices occur without sacrificing the probing frequency.
This will help mitigate the dependency of the speed of system response on any single communication channel.

Here, the central entity collects objective function measurements over a time horizon and, and computes a batch (i.e. a vector) of measurements. The controllers receives the broadcast batch of objectives and process it at once, computing an averaged gradient estimate used to move the ESC setpoint. Additionally, the reader should note that in this formulation, the distributed controller can improve the demodulation stage of the ES algorithm, by proceeding aligning the demodulation signal by autocorrelation.

By allowing measurements to collect and constructing a time series of objective values, then broadcasting the time series so that ESC resources can construct their gradients and new setpoints, we can decouple the measurement and probing speeds from the speed of the broadcast communications and control setpoint generation. This also allows the frequency of broadcast communications to drop dramatically, although the quantity of information in a broadcast increases commensurately.

We begin with an overview of the structure of batch 2D-ES (2D-BES) control scheme followed by simulation experiments that validate the approach. Concluding remarks are then provided. A stability analysis of the 2D-BES scheme is then presented in the appendix.

\section{Distribution Network Model and Objective Function Construction}

In this section, we formulate the objective function for the 2D-BES scheme. Consider an unbalanced distribution network with arbitrary topology. The set of nodes is denoted by $\mathcal{N}$, and the set of lines that connect nodes is denoted by $\mathcal{L}$.

Kirchoff's Voltage Law (KVL) expressed for adjacent nodes $m$ and $n$ connected by line $(m, n)$ is:

$$
\left[\boldsymbol{e}_{m} \circ \angle \boldsymbol{\delta}_{m}=\boldsymbol{e}_{n} \circ \angle \boldsymbol{\delta}_{n}+\boldsymbol{Z}_{m n} \boldsymbol{i}_{m n}\right]_{\mathcal{P}_{m n}},
$$

where $\boldsymbol{e}_{m}=\left[e_{m}^{a} \angle 0, e_{m}^{b} \angle 0, e_{m}^{c} \angle 0,\right]^{T}$ is the vector of node voltage phasor magnitudes at node $m, \boldsymbol{\delta}_{m}=\left[\delta_{m}^{a}, \delta_{m}^{b}, \delta_{m}^{c},\right]^{T}$ is the vector of votlage phase angles at node $m, \boldsymbol{e}_{m} \circ \angle \boldsymbol{\delta}_{m}$ is the complex vector of voltage phasors at node $m$ where $\angle \boldsymbol{\delta}_{m}=\left[1 \angle \delta_{m}^{a}, \angle 1 \delta_{m}^{b}, 1 \angle \delta_{m}^{c}\right]^{T} \in \mathbb{C}^{3 \times 1}$, $\boldsymbol{Z}_{m n}$ is the impedance matrix for line $(m, n)$, $\boldsymbol{i}_{m n}=\left[i_{m n}^{a}, i_{m n}^{b}, i_{m n}^{c},\right]^{T}$ is the vector of node current phasors on line $(m, n)$ from node $m$ to node $n$. The notation $[\cdot]_{\mathcal{P}_{m n}}$ indexes the equation by the set 
of phases $\left(\mathcal{P}_{m n}\right)$ of line $(m, n)$, as in [8,9]. It should be noted that $\boldsymbol{e}_{m}^{\phi}=\left|\boldsymbol{e}_{m}^{\phi} \angle \delta_{m}^{\phi}\right|$ and $\delta_{m}^{\phi}=\angle\left(\boldsymbol{e}_{m}^{\phi} \angle \delta_{m}^{\phi}\right)$ where the superscript $\phi$ denotes an arbitrary phase.

Kirchoff's Current Law (KCL) for node $m$ is written as:

$$
\boldsymbol{i}_{m}+\sum_{n:(m, n) \in \mathcal{L}}\left[\boldsymbol{i}_{m n}\right]_{\mathcal{P}_{m n}}=0
$$

where $\boldsymbol{i}_{m}=\left[i_{m}^{a}, i_{m}^{b}, i_{m}^{c},\right]^{T}$ denotes the current entering node $m$. We take the Hadamard Product of $\boldsymbol{e}_{m}$ 。 $\angle \boldsymbol{\delta}_{m}$ with the complex conjugate of (2) and substitue for $\boldsymbol{e}_{m} \circ \angle \boldsymbol{\delta}_{m}$ with (1) inside the summation, giving:

$$
\begin{aligned}
& \boldsymbol{e}_{m} \circ \angle \boldsymbol{\delta}_{m} \circ \boldsymbol{i}_{m}^{*} \ldots \\
& +\sum_{n:(m, n) \in \mathcal{L}}\left[\left(\boldsymbol{e}_{n} \circ \angle \boldsymbol{\delta}_{n}+\boldsymbol{Z}_{m n} \boldsymbol{i}_{m n}\right) \circ \boldsymbol{i}_{m n}^{*}\right]_{\mathcal{P}_{m n}}=0 .
\end{aligned}
$$

This can be rewritten as in terms of node loads, node power injections, and line complex power flows and line losses as:

$$
\boldsymbol{d}_{m}+\boldsymbol{\mu}_{m}+\sum_{n:(m, n) \in \mathcal{L}}\left[\left(\boldsymbol{s}_{m n}+\boldsymbol{l}_{m n}\right)\right]_{\mathcal{P}_{m n}}=0
$$

where $\boldsymbol{d}_{m}=\left[d_{m}^{a}, d_{m}^{b}, d_{m}^{c},\right]^{T}$ is the vector of complex loads (including capacitors), $\boldsymbol{\mu}_{m}=\left[\mu_{m}^{a}, \mu_{m}^{b}, \mu_{m}^{c},\right]^{T}$ is the vector of complex power injections (due to DER), $\boldsymbol{s}_{m n}=\left[s_{m n}^{a}, s_{m n}^{b}, s_{m n}^{c},\right]^{T}$ is the vector of complex power phasors for line $(m, n)$ entering node $n$, and $\boldsymbol{l}_{m n}=\left[l_{m n}^{a}, l_{m n}^{b}, l_{m n}^{c},\right]^{T}$ is the vector of complex power losses on line $(m, n)$.

Finally, we can rewrite (1) by substituting line current with line power divided by node voltage:

$\left[\boldsymbol{e}_{m} \circ \angle \boldsymbol{\delta}_{m}=\boldsymbol{e}_{n} \circ \angle \boldsymbol{\delta}_{n}+\boldsymbol{Z}_{m n} \boldsymbol{s}_{m n} \oslash\left(\boldsymbol{e}_{n} \circ \angle \boldsymbol{\delta}_{n}\right)\right]_{\mathcal{P}_{m n}}$,

where $\oslash$ denotes Hadamard division (index-wise division). For a full discussion of how to explicity define voltage phasor magnitude and voltage phasor angle from (4) and (5), the reader is invited to view [9].

Using the model outlined in Eqs (1)-(5), we formulate the following Optimal Power Flow problem to track real and reactive power targets at the distribution substation while performing feeder voltage regulation:

$$
\begin{array}{cc}
\underset{\substack{u_{m}^{\phi}, v_{m}^{\phi} \\
e_{m}^{\phi}, P_{m n}^{\phi}, Q_{m n}^{\phi}}}{\operatorname{minimize}} & \sum_{\phi \in\{a, b, c\}} \alpha_{P}^{\phi}\left(P_{\infty, 0}^{\phi}-P_{t}^{\phi}\right)^{2}+\ldots \\
& \sum_{\phi \in\{a, b, c\}} \alpha_{Q}^{\phi}\left(Q_{\infty, 0}^{\phi}-Q_{t}^{\phi}\right)^{2}
\end{array}
$$

subject to: $\quad(1)-(5)$,

$$
\begin{aligned}
& \forall \phi \in \mathcal{P}_{m}, \forall m \in \mathcal{N} \\
& \underline{e}_{m}^{\phi} \leq e_{m}^{\phi} \leq \bar{e}_{m}^{\phi} \\
& \underline{u}_{m}^{\phi} \leq u_{m}^{\phi} \leq \bar{u}_{m}^{\phi}, \\
& \underline{v}_{m}^{\phi} \leq v_{m}^{\phi} \leq \bar{v}_{m}^{\phi}, \\
& \underline{w}_{m}^{\phi} \leq \sqrt{\left(u_{m}^{\phi}\right)^{2}+\left(v_{m}^{\phi}\right)^{2}} \leq \bar{w}_{m}^{\phi}, \\
& \forall \phi \in \mathcal{P}_{m n}, \forall(m, n) \in \mathcal{L} \\
& \underline{P}_{m n}^{\phi} \leq P_{m n}^{\phi} \leq \bar{P}_{m n}^{\phi}, \\
& \underline{Q}_{m n}^{\phi} \leq Q_{m n}^{\phi} \leq \bar{Q}_{m n}^{\phi}, \\
& \underline{S}_{m n}^{\phi} \leq \sqrt{\left(P_{m n}^{\phi}\right)^{2}+\left(Q_{m n}^{\phi}\right)^{2}} \leq \bar{S}_{m n}^{\phi},
\end{aligned}
$$

where $P_{t}^{\phi}$ and $Q_{t}^{\phi}$ are the substation active and reactive power targets, $\alpha_{P}^{\phi}, \alpha_{Q}^{\phi}$ are scaling factors. Without loss of generality, we have assumed the existence of a four quadrant-capable DER at every node in $\mathcal{N} \backslash\{\infty, 0\}$.

Let the individual constraints in (6) be represented by the vector $\phi(\boldsymbol{y}, \boldsymbol{P}, \boldsymbol{Q}, \boldsymbol{\mu}) \in \mathcal{R}^{d \times 1}$ (i.e. we have $d$ inequality constraints), with $\boldsymbol{\mu}=\left[u_{1}, \ldots, u_{N}, v_{1}, \ldots, v_{N}\right]^{T}$. In order to utilize 2D-BES control, we transform the problem (6) into an equivalent form via making successive substitutions of the equality constraints into the inequality constraints and the objective function, and, subsequently, approximating the inequality constraints with penalty functions in the objective, resulting in:

$$
\underset{\boldsymbol{\mu}}{\operatorname{minimize}} \psi(\boldsymbol{\mu}),
$$

where

$$
\begin{aligned}
\psi(\boldsymbol{\mu})= & \alpha_{P}^{\phi}\left(P_{\infty, 0}^{\phi}(\boldsymbol{\mu})-P_{t}^{\phi}\right)^{2} \\
& +\alpha_{Q}^{\phi}\left(Q_{\infty, 0}^{\phi}(\boldsymbol{\mu})-Q_{t}^{\phi}\right)^{2} \\
& +\lambda_{m} \sum_{m=0}^{N} h(\phi(\boldsymbol{\mu}))
\end{aligned}
$$


and $h\left(\phi_{m}(\boldsymbol{\mu})\right)=\max \left(0, \phi_{m}(\boldsymbol{\mu})\right)^{2}$, with $\lambda_{m}$ positive. The last term of (8) is a generalized penalty function to enforce proper limits on voltages and line flows. We note that the local convexity of (8) was established in [5].

\section{Batch Extremum Seeking Control}

In this section, we present the 2D-BES algorithm. We consider an Extremum Seeking algorithm in which the objective function measurements are not provided to ES controllers in real time. Instead, objective function measurements are provided to the controllers in batches at unknown and uneven intervals. The batch information is broadcast by a central entity to all 2D-BES controllers, which then independently process the information and update their DER setpoints.

This approach assumes that measurements collected by the central entity are timestamped. As the batch of measurements will be broadcast to controllers after the measurement period, the controllers need to synchronize the objective function measurements with past perturbations.

An overview of the 2D-BES approach is shown in Figure 1. The approach consists of three processes: system perturbation (SP), gradient estimation (GE), and setpoint update (SU). During the SP phase, the 2D-BES controllers command DER to modulate their active and reactive power contributions sinusoidally. We refer to this process as "probing" or "probe injection". These sinusoial power injections cause sinusoidal oscillations in system voltage and power flows. A central entity then measures voltages and power flows at locations where control action is desired and, at each timestep, computes a scalar value assessing the effectiveness of the control action (i.e. an OPF objective function). In the 2D-BES scheme, we assume that the central entity collects and stores measurements of the objective function and broadcasts a brief history of past objective function values (i.e. a "batch" of information) to the 2D-BES controllers.

Once a 2D-BES controller receives a batch, it begins the GE process to obtain an estimate of the gradient of the objective function with respect to their control inputs over the batch time period. Next, the 2D-BES controllers update their setpoints based on the newly computed gradient estimates, in order to minimize the objective function. The SU process may overlap part of the next batch, and the next batch may contain measurements when one or more 2D-BES controller is updating their setpoints.

We now examine in detail the three processes that constitute 2D-BES, but do not discuss stability in this section. An investigation into the stability of this approach is found in the appendix of this work.

Let the batches of measurements be indexed by $b$, and denote the start time of the $b^{\text {th }}$ batch as $t_{b}$, and the end time of the $b^{t h}$ batch as $\overline{t_{b}}$. The objective function (see (8)) during this period is $\psi(\boldsymbol{\mu}(t)), t \in\left[\underline{t_{b}}, \overline{t_{b}}\right]$. A centralized entity collects values of $\psi(\boldsymbol{\mu}(t))$, and broadcasts this batch at a time after $\overline{t_{b}}$.

We acknowledge the possibility for the placement of multiple 2D-BES controllers at a node, or the placement of multiple 2D-BES controllers on the same phase at a node. Therefore, we modify our nomenclature slightly when discussing 2D-BES controllers. A single subscript will denote the controller index, and not its node. In a double subscript, the first part will denote the active, or reactive, power loop of the 2D-BES controller by $u$, or $v$, respectively. We do not utilize a superscript to the network phase of the controller.

\subsection{System Perturbation}

During the SP process, the 2D-BES controllers probe the system with a sinusoidal perturbation signal. Each 2D-BES adds a sinusoidal perturbation to its active power setpoint, $\hat{u}_{m}$, and a sinusoidal perturbation offset by $\pi / 2$ to its reactive power setpoint, as in (9) - (10):

$$
\begin{aligned}
& u_{m}(t)=\hat{u}_{m}(t)+a_{u, m}(t) \cos \left(\omega_{m} t\right), \\
& v_{m}(t)=\hat{v}_{m}(t)+a_{v, m}(t) \sin \left(\omega_{m} t\right) .
\end{aligned}
$$

We define $\boldsymbol{\mu}(t)$ as the vector of all active and reactive power injections, $\hat{\boldsymbol{\mu}}(t)$ as the vector of all active and reactive power setpoints, $\boldsymbol{A}$ as the diagonal matrix of active and reactive power probe amplitudes, and $\boldsymbol{r}(t)$ as the vector of active and reactive power, in (11) - (14):

$$
\begin{gathered}
\boldsymbol{\mu}(t)=\left[u_{1}(t), \ldots, u_{n}(t), v_{1}(t), \ldots v_{n}(t)\right]^{T}, \\
\hat{\boldsymbol{\mu}}(t)=\left[\hat{u}_{1}(t), \ldots, \hat{u}_{n}(t), \hat{v}_{1}(t), \ldots, \hat{v}_{n}(t)\right]^{T}, \\
\boldsymbol{A}=\operatorname{diag}\left(\left[a_{u, 1}, \ldots, a_{u, n}, a_{v, 1}, \ldots, a_{v, n}\right]^{T}\right), \\
\boldsymbol{r}(t)=\left[\cos \left(\omega_{1} t\right), \ldots, \cos \left(\omega_{n} t\right), \ldots\right. \\
\left.\sin \left(\omega_{1} t\right), \ldots, \sin \left(\omega_{n} t\right)\right]^{T},
\end{gathered}
$$

and express (9) and (10) for all 2D-BES controllers in compact form as:

$$
\boldsymbol{\mu}(t)=\hat{\boldsymbol{\mu}}(t)+\boldsymbol{A} \boldsymbol{r}(t) .
$$

The 2D-BES controllers inject their active and reactive power probes into the network. A central entity records and timestamps measurements of the objective 


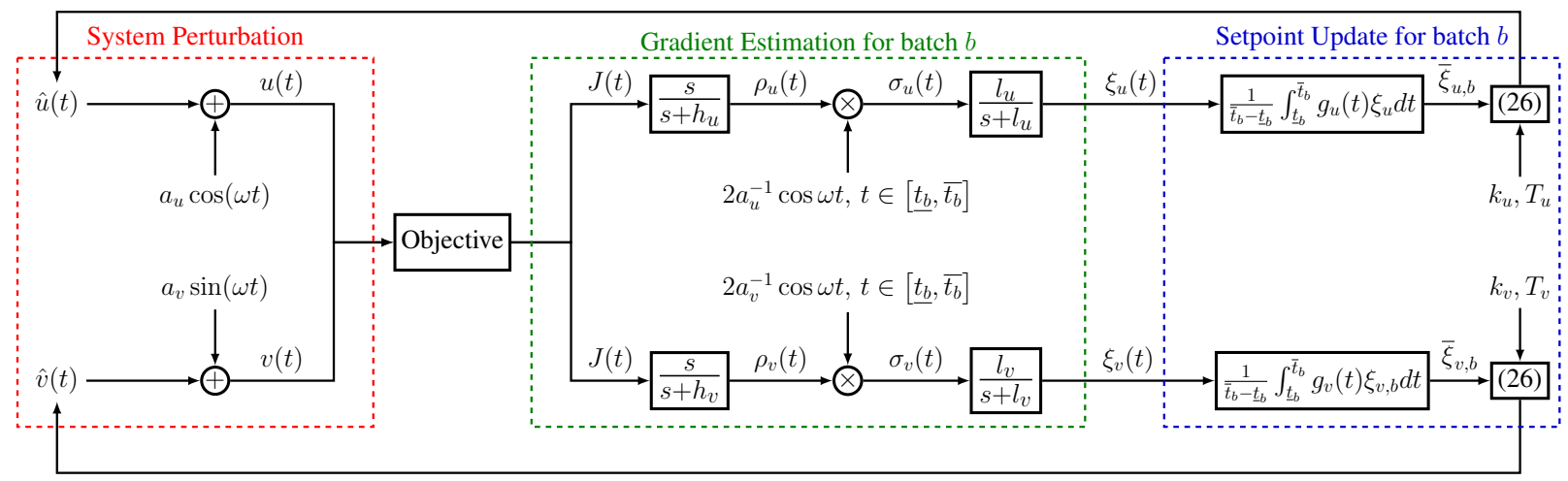

Figure 1. 2D-BES control loop block diagram for a single controller.

function $\psi(\boldsymbol{\mu}(t))$. The central entity broadcasts the objective function measurements for the $b^{\text {th }}$ batch from the period $t \in\left[t_{b}, \overline{t_{b}}\right]$ to all controllers at a time $t \geq$ $t_{b}$. At this point, the 2D-BES controller begin the GE process.

The 2D-BES algorithm relies the ability of each controller, to estimate the gradient of the objective function with respect to its setpoint only. The present work utilizes sinusoidal probing signals, though extremum seeking allows other types of probes (triangle and square waves, for example, even stochastic processes may be used as probes). When utilizing sinusoidal probes on multiple controllers, two criteria must be satisfied for the scheme to work:

$$
\begin{aligned}
\omega_{m} & \neq \omega_{n} & \forall m, n & \in \mathcal{C} \\
\omega_{k}+\omega_{m} & \neq \omega_{n} & \forall k, m, n & \in \mathcal{C}
\end{aligned}
$$

The active and reactive power loops of a single controller may use the same frequency, only if the probes are offset by $\pi / 2$.

\subsection{Gradient Estimation}

Once a 2D-BES controller receives objective measurements for batch $b$ over the period $t \in$ $\left[t_{b}, \overline{t_{b}}\right]$, it processes the measurements to obtain a gradient estimate over the batch time horizon. Due to possible noncontiguity of batch reporting, the 2D-BES controllers do not take any information from previous batches into account while processing the current batch, and initialize all internal states associated with the GE process as 0 . The reader should note that this approach is designed to be robust to missing measurements and delays in measurement broadcast.

For clarity and to examine the approach in detail, we now focus on the active power channel of the $m^{\text {th }}$ 2D-BES controller, which is depicted in Figure 1. First, the objective function passes through a high-pass filter, to attenuate low frequency content of the objective function. Using a first-order Taylor Expansion, the objective function can be expressed over the period $t \in\left[\underline{t_{b}}, \overline{t_{b}}\right]$ as:

$$
\begin{aligned}
& \psi(\boldsymbol{\mu}(t)) \approx \psi(\hat{\boldsymbol{\mu}}(t))+\nabla_{\boldsymbol{\mu}} \psi(\hat{\boldsymbol{\mu}}(t))(\boldsymbol{\mu}(t)-\hat{\boldsymbol{\mu}}(t)) \\
& \psi(\boldsymbol{\mu}(t)) \approx \psi(\hat{\boldsymbol{\mu}}(t))+\nabla_{\boldsymbol{\mu}} \psi(\hat{\boldsymbol{\mu}}(t)) \boldsymbol{A r}(t) .
\end{aligned}
$$

The high-pass filter attenuates $\psi(\hat{\boldsymbol{\mu}}(t))$, such that the output is $\rho_{u, m}(t), t \in\left[\underline{t_{b}}, \overline{t_{b}}\right]$ can be approximated as:

$$
\begin{gathered}
\rho_{u, m}(t) \approx \nabla_{\boldsymbol{\mu}} \psi(\hat{\boldsymbol{\mu}}(t)) \boldsymbol{A r}(t), \\
\rho_{u, m}(t) \approx \sum_{n=1}^{N} \nabla_{u_{n}} \psi(\hat{\boldsymbol{\mu}}(t)) a_{u, n} \cos \left(\omega_{n} t\right) \\
+\sum_{n=1}^{N} \nabla_{v_{n}} \psi(\hat{\boldsymbol{\mu}}(t)) a_{v, n} \sin \left(\omega_{n} t\right),
\end{gathered}
$$

which shows how the objective function is changing with respect to the probing perturbations.

Second, the output of the high-pass filter is multiplied by the appropriate demodulation signal giving $\sigma_{u, m}(t), t \in\left[\underline{t_{b}}, \overline{t_{b}}\right]$ :

$$
\begin{aligned}
\sigma_{u, m}(t) \approx & \frac{2}{a_{u, m}} \cos \left(\omega_{m} t\right) \nabla_{\boldsymbol{\mu}} \psi(\hat{\boldsymbol{\mu}}(t)) \boldsymbol{A} \boldsymbol{r}(t), \\
\sigma_{u, m}(t) \approx & \frac{2 \cos \left(\omega_{m} t\right)}{a_{u, m}} \sum_{n=1}^{N} \nabla_{u_{n}} \psi(\hat{\boldsymbol{\mu}}) a_{u, n} \cos \left(\omega_{n} t\right) \\
& +\frac{2 \cos \left(\omega_{m} t\right)}{a_{u, m}} \sum_{n=1}^{N} \nabla_{v_{n}} \psi(\hat{\boldsymbol{\mu}}) a_{v, n} \sin \left(\omega_{n} t\right) .
\end{aligned}
$$

Third, the demodulated signal $\sigma_{u, m}(t)$ passes through a low-pass filter to attenuate oscillatory 
components. By the orthogonality of sinusoidal functions, the only term with a static component is $2 \nabla_{u_{m}} \psi(\hat{\boldsymbol{\mu}}(t)) \cos ^{2}\left(\omega_{m} t\right), t \in\left[t_{b}, \overline{t_{b}}\right]$. Therefore the output of the low-pass filter is an estimate of the gradient of the objective function with respect to the active power setpoint over the period $t \in\left[\underline{t_{b}}, \overline{t_{b}}\right]$ :

$$
\xi_{u, m}(t) \approx \nabla_{u_{m}} \psi(\hat{\boldsymbol{\mu}}(t)) .
$$

The reader should note that this gradient estimate is in the "average sense", which is explained in detail in the appendix.

\subsection{Setpoint Update}

After a 2D-BES controller obtains a gradient estimate for the period $t \in\left[t_{b}, \overline{t_{b}}\right]$, it updates its real and reactive power setpoints. There are many ways in which the setpoints $\hat{u}_{m}$ and $\hat{v}_{m}$ can be updated, and will likely be subject to hardware and system constraints. In our current approach, we calculate a weighted average of the gradient estimate for the $b^{t h}$ batch to update $\hat{u}_{m}$ and $\hat{v}_{m}$. We denote the weighted average for the active power channel of the $m^{t h} 2 \mathrm{D}-\mathrm{BES}$ controller as $\bar{\xi}_{u, m, b}$, defined as:

$$
\bar{\xi}_{u, m, b}=\frac{1}{\overline{t_{b}}-\underline{t_{b}}} \int_{\underline{t_{b}}}^{\overline{t_{b}}} g(t) \xi_{u, m}(t) d t .
$$

The active power setpoint is then linearly updated over the period $T_{u, m}$ after $\overline{t_{b}}$, and held constant afterwards until the next batch of measurements is received, such that:

$$
\hat{u}_{m}(t)=\left\{\begin{array}{c}
\hat{u}_{m}\left(\overline{t_{b}}\right)-k_{u, m} T_{u, m}^{-1} \bar{\xi}_{b, u, m}\left(t-\overline{t_{b}}\right) \\
t \in\left[\overline{t_{b}}, \overline{t_{b}}+T_{u, m}\right] \\
\hat{u}_{m}\left(\overline{t_{b}}+T_{u, m}\right), t \geq \overline{t_{b}}+T_{u, m}
\end{array}\right.
$$

with $k_{m}>0 \forall m \in \mathcal{C}$.

The reader should note that the SU step from batch $b$ may overlap with batch $b+1$. This does not affect the stability of the 2D-BES algorithm, and can be accounted for in several ways. First, the high-pass filter attenuates portions of the objective function that correspond to the setpoint and the update after batch $b$. Second, portions of batches that contain setpoint updates from the previous batch can be discarded from the GE process, or thirdly, omitted from the SU process by choice of $g_{u}(t)$ and $g_{v}(t)$.

\section{Simulations}

To investigate the behavior of 2D-BES control, we conducted simulations in which a group of DER, each

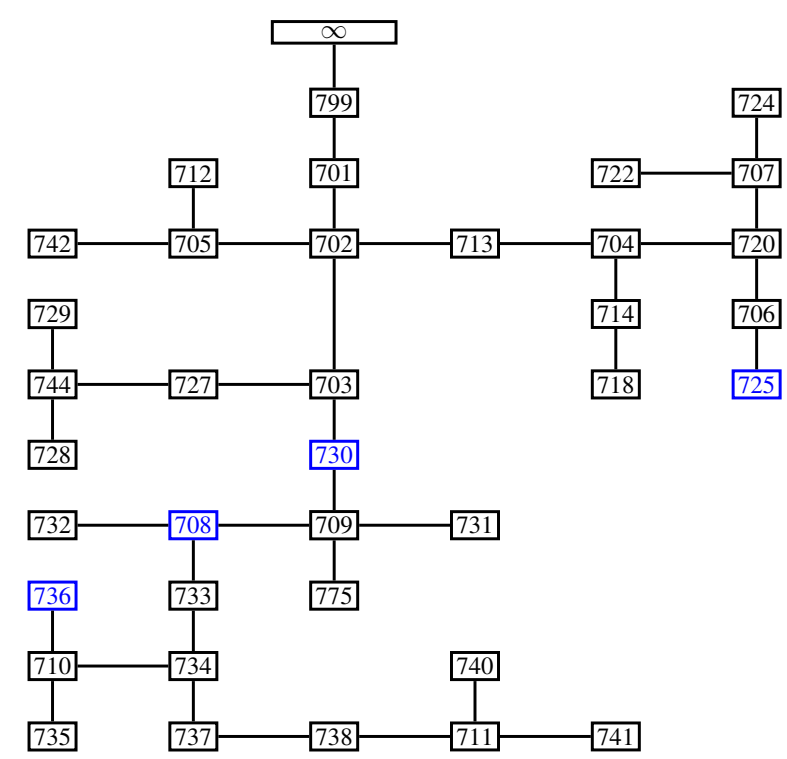

Figure 2. IEEE 37 node test feeder topology. Nodes with DER are blue.

controlled by a 2D-BES algorithm, dispatched their active and reactive power injections to track both active power and reactive power targets at the substation. The objective function of the simulation is:

$$
\begin{aligned}
\psi(\boldsymbol{\mu}(t)) & =\sum_{\phi \in\{a, b, c\}} \alpha_{P}\left(P_{\infty, 799}^{\phi}(\boldsymbol{\mu}(t))-P_{t}^{\phi}(t)\right)^{2} \cdots \\
& +\sum_{\phi \in\{a, b, c\}} \alpha_{Q}\left(Q_{\infty, 799}^{\phi}(\boldsymbol{\mu}(t))-Q_{t}^{\phi}(t)\right)^{2}
\end{aligned}
$$

with coefficients of $\alpha_{P}=\alpha_{Q}=1$. In this simulation, we assume that the group of DER are dispatched to minimize a common objective function. Objective function measurements are broadcast to all 2D-BES controllers.

Simulations were conducted on a modified version of the IEEE 37 node test feeder, the topology of which can be seen in Figure 2. The voltage regulator between nodes 799 and 701 was omitted, and the switch between 709 and 775 was replaced by a line with configuration 724 and length of $15.25 \mathrm{~m}$. All loads were multiplied by a factor of 1.5, and were assumed to follow a ZIP model of the form:

$$
\begin{aligned}
& p_{m}^{\phi}\left(y_{m}^{\phi}\right)=\left(\beta_{m, S}^{\phi}+\beta_{m, I}^{\phi}\left(y_{m}^{\phi}\right)^{\frac{1}{2}}+\beta_{m, Z}^{\phi} y_{m}^{\phi}\right) \cdot p_{m}^{\phi}, \\
& q_{m}^{\phi}\left(y_{m}^{\phi}\right)=\left(\beta_{m, S}^{\phi}+\beta_{m, I}^{\phi}\left(y_{m}^{\phi}\right)^{\frac{1}{2}}+\beta_{m, Z}^{\phi} y_{m}^{\phi}\right) \cdot q_{m}^{\phi},
\end{aligned}
$$

with parameters: $\beta_{m, S}^{\phi}=0.75, \beta_{m, I}^{\phi}=0.10, \beta_{m, Z}^{\phi}=$ 
$0.15 \forall \phi \in\{a, b, c\} \forall m \in \mathcal{N}$

DER were placed on all phases at nodes 725, 730, 708, and 736. All DER were assumed to be capable of four-quadrant and phase-independent operation. Each DER had its own 2D-BES controller for simultaneous management of active and reactive power. The same values for each parameter were assigned to both the active and reactive power control loops, and therefore we omit the subscripts of $u$ and $v$ when discussing parameters. Controller node, phase and frequency generation parameter $p_{m}$ are listed in Table 1. Controller probe frequencies were assigned as $f_{m}=0.1 \sqrt{p_{m}} /\left\lfloor\sqrt{p_{m}}\right\rfloor$. Probe amplitudes were 0.003 p.u. High-pass filter cutoff frequencies were assigned as $h_{m}=2 \pi f_{m} / 10$, and low-pass filter cutoff frequencies were assigned as $l_{m}=2 \pi f_{m} / 10$. The gradient weighting functions were assigned to all controllers as $g_{m}(t)=1$. The update times were assigned to all controllers as $T_{m}=15$. The gain for all active power controller loops was 0.005 , and the gain for all reactive power controllers loops was 0.0025 .

All batches were 60 seconds long. Gaps between batches had durations of a random integer number of seconds. We assume that objective function measurements were broadcast to the 2D-ES controllers at the end of each batch. Table 2 lists the active, and reactive, power targets for this experiment.

Figure 3 plots the objective function, substation active and reactive power, and the associated substation power targets for each phase. The 2D-BES controllers successfully manage their respective power to converge the substation active power, and reactive power, to their respective targets. The $2 \mathrm{D}-\mathrm{BES}$ controllers respond to a change in the targets at 900 seconds, and track the new targets.

Figure 4 shows the gradient estimates, averaged gradient estimates, and active power setpoints for the active power channels of the 2D-BES controllers at node 736, with the gradient estimates and average gradient estimates only plotted for batch periods. Figure 5 shows the gradient estimates, averaged gradient estimates, and reactive power setpoints for the reactive power channels of the 2D-BES controllers at node 736, with the gradient estimates and average gradient estimates only plotted for batch periods.

In this experiment, batches contained 60 seconds of contiguous measurements. In a practical implementation, batch length will likely be determined by hardware and communication system limitations. However, we investigated the effect of batch length on the performance of the 2D-BES algorithm, and observed a tradeoff between the accuracy gradient estimate and convergence speed. Longer batches provide better
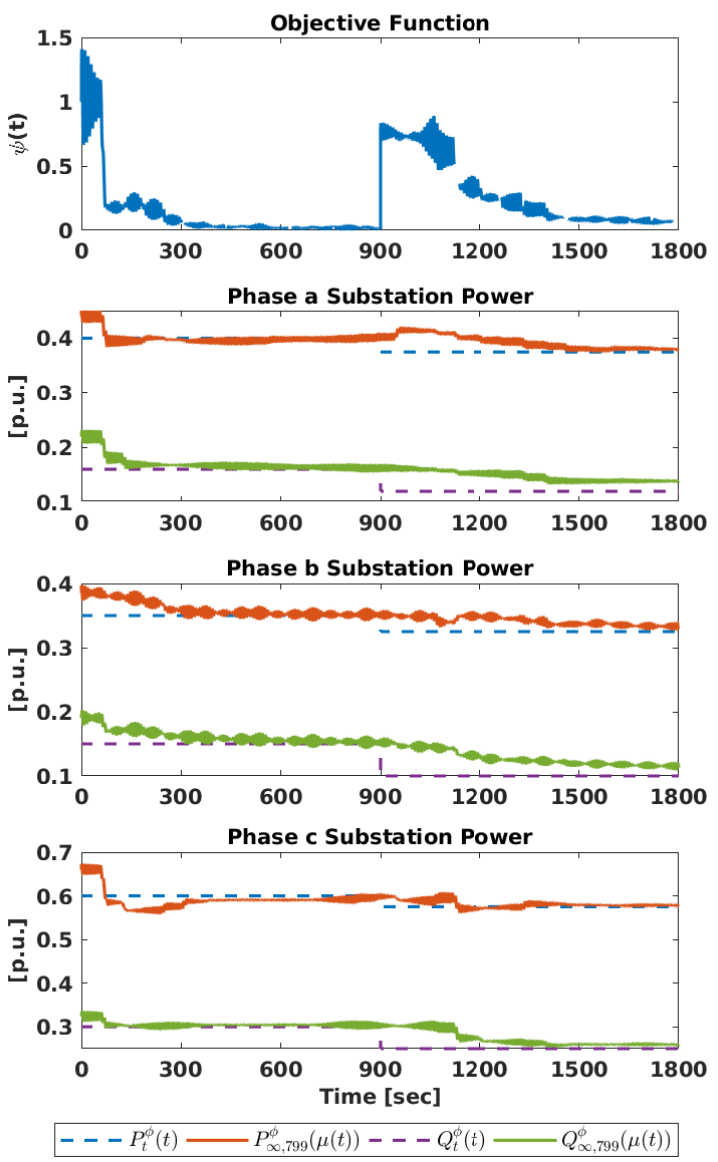

Figure 3. Objective function, and per phase substation active and reactive powers, and per phase substation active and reactive power references. The objective function is only plotted for batch periods, and has been normalized by the first measured value.

gradient estimates, as transient components will be averaged out. However, the lower setpoint update frequency slows convergence speed, and reduces the ability of the controllers to respond to rapid changes in loads or optimization targets.

\section{Conclusions}

This work developed an extension to previously studied 2-dimensional Extremum Seeking (2D-ES) control algorithms to accommodate for communication delays and information loss that seem to naturally occur in actual physical deployments. To mitigate these issues, we proposed a "batch" Extremum Seeking approach that partitioned the 2D-BES algorithm into distinct gradient estimation and update steps. Breaking up the 
Table 1. 2D-BES controller parameters, including node, phase, and prime number for frequency generation.

\begin{tabular}{|l|c|c|c|c|c|c|c|c|c|c|c|c|}
\hline Controller & 1 & 2 & 3 & 4 & 5 & 6 & 7 & 8 & 9 & 10 & 11 & 12 \\
Node & 705 & 705 & 705 & 725 & 725 & 725 & 725 & 725 & 725 & 731 & 731 & 731 \\
Phase & $\mathrm{a}$ & $\mathrm{b}$ & $\mathrm{c}$ & $\mathrm{a}$ & $\mathrm{b}$ & $\mathrm{c}$ & $\mathrm{a}$ & $\mathrm{b}$ & $\mathrm{c}$ & $\mathrm{a}$ & $\mathrm{b}$ & $\mathrm{c}$ \\
$p_{m}$ & 11 & 13 & 17 & 19 & 23 & 29 & 31 & 37 & 41 & 43 & 47 & 53 \\
\hline
\end{tabular}
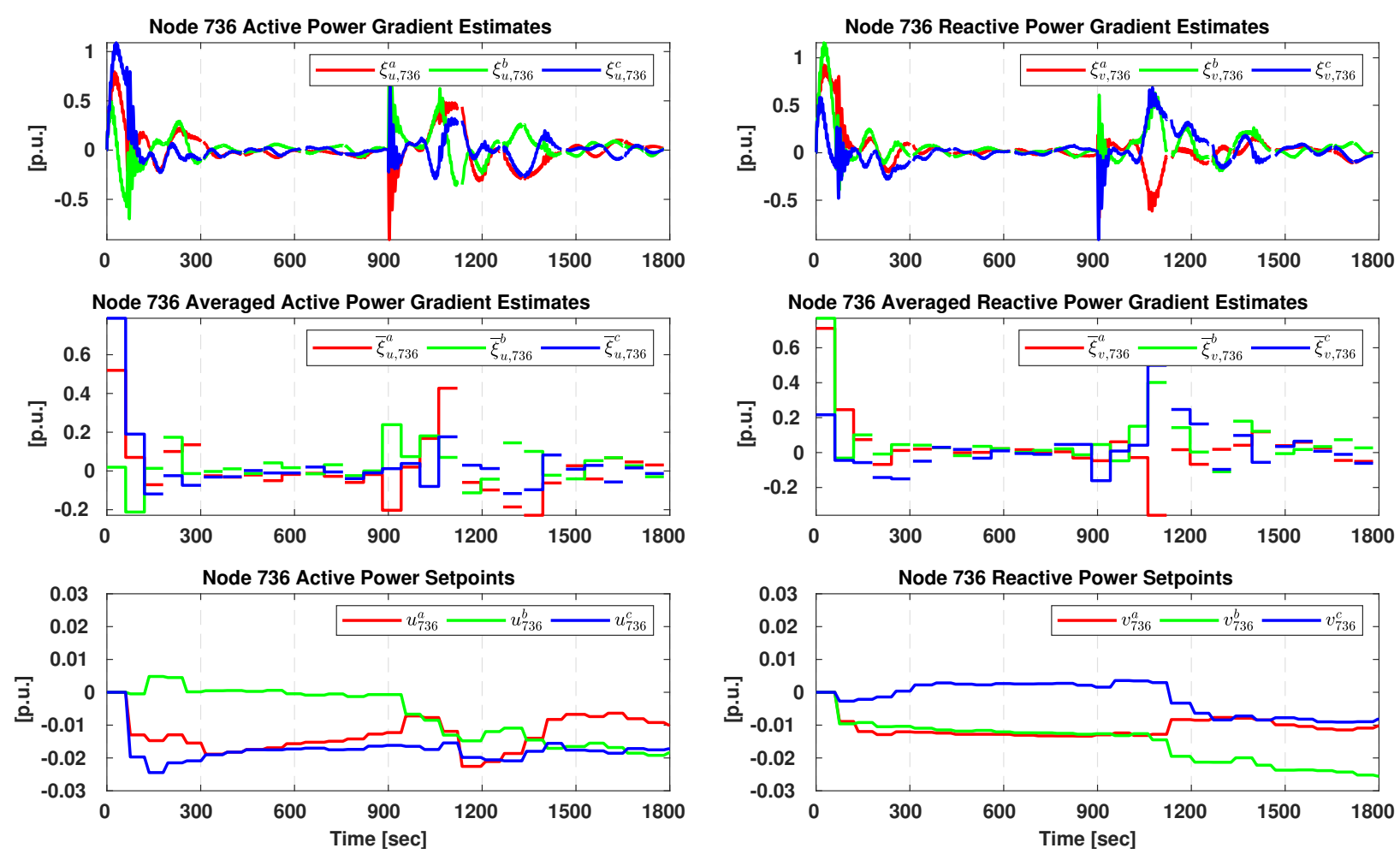

Figure 4. Gradient estimates, averaged gradient estimates, and setpoints for the active power channel of a 2D-BES controller at node 736 . Gradient estimate and average gradient estimate are only plotted for batch periods.

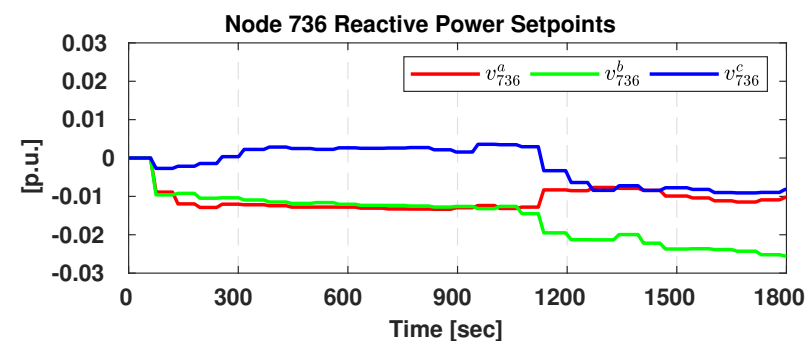

Figure 5. Gradient estimates, averaged gradient estimates, and setpoints for the reactive power channel of a 2D-BES controller at node 736. Gradient estimate and average gradient estimate are only plotted for batch periods.

Table 2. Substation active and reactive power targets.

\begin{tabular}{|l|c|c|c|}
\hline Time & $P_{t}^{a}$ & $P_{t}^{b}$ & $P_{t}^{c}$ \\
\hline$t \in[0,900]$ & 0.040 & 0.35 & 0.60 \\
$t \in[900,1800]$ & 0.375 & 0.325 & 0.575 \\
\hline Time & $Q_{t}^{a}$ & $Q_{t}^{b}$ & $Q_{t}^{c}$ \\
\hline$t \in[0,900]$ & 0.16 & 0.15 & 0.30 \\
$t \in[900,1800]$ & 0.14 & 0.125 & 0.275 \\
\hline
\end{tabular}

algorithm in this way allows for bulk processing of past objective function values, from which a gradient update rule is extracted that determines how to operate each DER until the next batch processing period. Like previous implementations of ES, the approach remains model-free and does not require global knowledge of

system power consumption. Although we demonstrated the efficacy of the approach to manage DER active and reactive power injections to track substation power targets, the approach generalizes to a wide variety of other objectives, including voltage and line power flow regulation, virtual islanding, and voltage phasor setpoint regulation. The improvement over our previous works made with batch 2D-BES stand to significantly improve the performance of the approach when deployed to physical systems. We plan to test the batch 2D-BES approach in hardware in the loop experiments in the future.

\section{References}

[1] Y. Zhang, M. Hong, E. Dall'Anese, S. Dhople, and Z. Xu, "Distributed controllers seeking ac optimal power flow 
solutions using admm," IEEE Trans. Smart Grid, vol. pp, pp. 1-13, 2017.

[2] Y. Xu, Z. Y. Dong, R. Zhang, and D. J. Hill, "Multi-timescale coordinated voltage/var control of high renewable-penetrated distribution systems," IEEE Trans. Power Syst., vol. 32, pp. 4398-4408, Nov. 2017.

[3] K. E. Antoniadou-Plytaria, I. N. Kouveliotis-Lysikatos, P. S. Georgilakis, and N. D. Hatziargyriou, "Distributed and decentralized voltage control of smart distribution networks: Models, methods, and future research," IEEE Trans. Smart Grid, vol. 8, pp. 2999-3008, Nov. 2017.

[4] M. Krstic and H. Wang, "Stability of extremum seeking feedback for general nonlinear dynamic systems," Automatica, vol. 36, no. 4, pp. 595 - 601, 2000.

[5] D. B. Arnold, M. D. Sankur, M. Negrete-Pincetic, and D. Callaway, "Model-free optimal coordination of distributed energy resources for provisioning transmission-level services," IEEE Trans. Power Syst., vol. 33, pp. 817-829, Jan. 2018

[6] M. Sankur and D. Arnold, "Extremum seeking control of distributed energy resources with decaying dither and equilibrium-based switching," in Proceedings of the 52nd Hawaii International Conference on System Sciences, 2019.

[7] J. Johnson, A. Summers, R. Darbali-Zamora, J. Hernandez-Alvidrez, J. Quiroz, D. Arnold, and J. Anandan, "Distribution voltage regulation using extremum seeking control with power hardware-in-the-loop," IEEE Journal of Photovoltaics, vol. 8, pp. 1824-1832, Nov 2018.

[8] E. Dall'Anese, H. Zhu, and G. Giannakis, "Distributed optimal power flow for smart microgrids," IEEE Trans. Smart Grid, vol. 4, no. 3, pp. 1464-1475, 2013.

[9] M. Sankur, Optimal Control of Commercial Office Battery Systems, and Grid Integrated Energy Resources on Distribution Networks. PhD thesis, UC Berkeley, 2017.

\section{Appendix}

We now examine the stability of the batch 2D-BES approach. We first present a continuous-time stability analysis of the GE for batch $b$ over the period $t \in$ $\left[t_{b}, \overline{t_{b}}\right]$. All variables in this analysis are for this period only, and thus, we will omit the $(t)$ notation. First, we introduce the following definitions:

$$
\begin{aligned}
\boldsymbol{\rho} & =\left[\rho_{u, 1}, \ldots, \rho_{u, N}, \rho_{v, 1}, \ldots, \rho_{v, N}\right]^{T} \\
\boldsymbol{\epsilon} & =\left[\epsilon_{u, 1}, \ldots, \epsilon_{u, N}, \epsilon_{v, 1}, \ldots, \epsilon_{v, N}\right]^{T} \\
\boldsymbol{\sigma} & =\left[\sigma_{u, 1}, \ldots, \sigma_{u, N}, \sigma_{v, 1}, \ldots, \sigma_{v, N}\right]^{T} \\
\boldsymbol{\xi} & =\left[\xi_{u, 1}, \ldots, \xi_{u, N}, \xi_{v, 1}, \ldots, \xi_{v, N}\right]^{T} \\
\boldsymbol{H} & =\operatorname{diag}\left(\left[h_{u, 1}, \ldots, h_{u, N}, h_{v, 1}, \ldots, h_{v, N}\right]^{T}\right), \\
\boldsymbol{L} & =\operatorname{diag}\left(\left[l_{u, 1}, \ldots, l_{u, N}, l_{v, 1}, \ldots, l_{v, N}\right]^{T}\right) \\
\boldsymbol{K} & =\operatorname{diag}\left(\left[k_{u, 1}, \ldots, k_{u, N}, k_{v, 1}, \ldots, k_{v, N}\right]^{T}\right)
\end{aligned}
$$

$$
\mathbf{1}=[1, \ldots, 1]^{T} \in \mathbb{R}^{2 N \times 1},
$$

where $\rho$ denotes the vector of high-pass filter outputs for all controllers, $\boldsymbol{\xi}$ denotes the vector of gradient estimates, $\boldsymbol{H}$ is a diagonal matrix of high-pass filter cutoff frequencies, $\boldsymbol{L}$ is a diagonal matrix of low-pass filter cutoff frequencies, $\boldsymbol{K}$ is a diagonal matrix of gains, and 1 is a vector of ones of dimension $2 n \times 1$. The vector $\epsilon$ denotes the vector of low-pass filtered objective function, defined as:

$$
\boldsymbol{\epsilon}=\psi(\boldsymbol{\mu}) \cdot \mathbf{1}-\boldsymbol{\rho}
$$

Next, we define $\phi$ as the vector containing all network squared voltage magnitudes, network voltage angles, network line active power flows, and network line reactive power flows. Let $\boldsymbol{\phi}=\boldsymbol{f}(\boldsymbol{\mu})$ denote the nonlinear mapping from $\boldsymbol{\mu} \rightarrow \phi$, where $\phi=\boldsymbol{f}(\boldsymbol{\mu})$ encompasses (1) - (5) for all nodes and lines in $\mathcal{G}$.

Again, without loss of generality, suppose the collection of 2D-BES controllers for a particular system are to be used to minimize a convex objective of the form:

$$
\psi=\left(\phi-\phi^{*}\right)^{T} \boldsymbol{M}\left(\phi-\phi^{*}\right),
$$

where $\boldsymbol{M}$ is a positive semidefinite and symmetric matrix (for $\psi$ to be convex), and $\phi^{*}$ is the optimizer of $\psi$. Consider a first order Taylor expansion of $\left(\phi-\phi^{*}\right)$ around the point $\boldsymbol{\mu}^{*}$ :

$$
\phi-\phi^{*}=\boldsymbol{f}(\boldsymbol{\mu})-\boldsymbol{f}\left(\boldsymbol{\mu}^{*}\right) \approx \boldsymbol{J}_{\mu}\left(\boldsymbol{\mu}^{*}\right)\left(\boldsymbol{\mu}-\boldsymbol{\mu}^{*}\right),
$$

where $\boldsymbol{J}_{\mu}(\cdot)=\nabla_{\boldsymbol{\mu}} \boldsymbol{f}(\cdot)$ is the Jacobian of $\boldsymbol{f}$ and is assumed to be full rank. Substituting (39) into (38) yields:

$$
\begin{aligned}
\psi & =\left(\boldsymbol{\mu}-\boldsymbol{\mu}^{*}\right)^{T} \underbrace{\boldsymbol{J}_{\mu}\left(\boldsymbol{\mu}^{*}\right)^{T} \boldsymbol{M} \boldsymbol{J}_{\mu}\left(\boldsymbol{\mu}^{*}\right)}_{\boldsymbol{B}}\left(\boldsymbol{\mu}-\boldsymbol{\mu}^{*}\right) \\
& =\left(\boldsymbol{\mu}-\boldsymbol{\mu}^{*}\right)^{T} \boldsymbol{B}\left(\boldsymbol{\mu}-\boldsymbol{\mu}^{*}\right) .
\end{aligned}
$$

The matrix $\boldsymbol{B}$ is positive semidefinite and symmetric due to the full rank of $\boldsymbol{J}_{\mu}\left(\boldsymbol{\mu}^{*}\right)$. We now analyze the stability of $N$ 2D-BES controllers in minimizing (41).

With (15) and $\tilde{\boldsymbol{\mu}}=\hat{\boldsymbol{\mu}}-\boldsymbol{\mu}^{*}$, we can rewrite (41) as:

$$
\psi(\tilde{\boldsymbol{\mu}}, \boldsymbol{r})=\tilde{\boldsymbol{\mu}}^{T} \boldsymbol{B} \tilde{\boldsymbol{\mu}}+2 \boldsymbol{r}^{T} \boldsymbol{A}^{T} \boldsymbol{B} \tilde{\boldsymbol{\mu}}+\boldsymbol{r}^{T} \boldsymbol{A}^{T} \boldsymbol{B} \boldsymbol{A} \boldsymbol{r}
$$

With (11) - (14) and (29) - (36), the dynamics of the high-pass filters, and low-pass filters, of all 2D-BES controllers are expressed compactly as (43) - (44):

$$
\begin{gathered}
\dot{\boldsymbol{\epsilon}}=\boldsymbol{H}(\psi(\tilde{\boldsymbol{\mu}}, \boldsymbol{r}) \cdot \mathbf{1}-\boldsymbol{\epsilon}) \\
\dot{\boldsymbol{\xi}}=2 \boldsymbol{L} \boldsymbol{A}^{-1} \boldsymbol{r} \circ(\psi(\boldsymbol{\mu}, \boldsymbol{r}) \cdot \mathbf{1}-\boldsymbol{\epsilon})-\boldsymbol{L} \boldsymbol{\xi}
\end{gathered}
$$


Let $\bar{\tau}$ be the lowest common multiple of all probe time periods, $\tau_{m}=2 \pi \omega_{m}^{-1}$, in the system such that the entire system is $\bar{\tau}$-periodic. The angular frequency of the dither of the $m^{t h}$ 2D-BES controller is: $\omega_{m}=\gamma_{m} \bar{\omega}$ where $\gamma_{m} \in \mathbb{Z}^{+} \backslash 0$, and $\bar{\omega}=2 \pi \bar{\tau}^{-1}$. We introduce the new timescale of $\tau=t \bar{\tau}^{-1}$, and can rewrite (43) and (44) as:

$$
\begin{aligned}
& \dot{\boldsymbol{\epsilon}}=\bar{\tau} \boldsymbol{H}(\psi(\tilde{\boldsymbol{\mu}}, \boldsymbol{r}) \cdot \mathbf{1}-\boldsymbol{\epsilon}) \\
& \dot{\boldsymbol{\xi}}=\bar{\tau}\left(2 \boldsymbol{L} \boldsymbol{A}^{-1} \boldsymbol{r} \circ(\psi(\boldsymbol{\mu}, \boldsymbol{r}) \cdot \mathbf{1}-\boldsymbol{\epsilon})-\boldsymbol{L} \boldsymbol{\xi}\right)
\end{aligned}
$$

where the dot notation on the left-hand side of (45) (46) now refers to $\frac{d}{d \tau}$, and all variables are functions of $\tau$. The system of equations (45) - (46) represent the 2D-BES dynamics of (43) - (44) in the new timescale over the period $\bar{\tau}$. This system is in the form to which averaging is applicable, and we integrate over the period 0 to $\bar{\tau}$, to obtain the "averaged" system dynamics of (47) $-(48)$ :

$$
\begin{gathered}
\dot{\boldsymbol{\epsilon}}_{a v}=\boldsymbol{H}\left(\left(\tilde{\boldsymbol{\mu}}_{a v}^{T} \boldsymbol{B} \tilde{\boldsymbol{\mu}}_{a v}+\frac{1}{2} \operatorname{Tr}\left(\boldsymbol{A}^{T} \boldsymbol{B} \boldsymbol{A}\right)\right) \cdot \mathbf{1}-\boldsymbol{\epsilon}_{a v}\right) \\
\dot{\boldsymbol{\xi}}_{a v}=\boldsymbol{L}\left(2 \boldsymbol{B} \tilde{\boldsymbol{\mu}}_{a v}-\boldsymbol{\xi}_{a v}\right)
\end{gathered}
$$

where the "av" subscript refers to $\frac{1}{\bar{\tau}} \int_{0}^{\bar{\tau}}(\cdot) d \tau$ and through the orthogonality of sinusoidal functions:

$$
\frac{1}{\bar{\tau}} \int_{0}^{\bar{\tau}} \boldsymbol{r} \boldsymbol{r}^{T} d \tau=\frac{1}{2}\left[\begin{array}{cc}
\boldsymbol{I}_{N \times N} & \mathbf{0}_{N \times N} \\
\mathbf{0}_{N \times N} & \boldsymbol{I}_{N \times N}
\end{array}\right] .
$$

As both $\boldsymbol{H}$ and $\boldsymbol{L}$ are diagonal and Hurwitz, the averaged system of (47) - (48) is stable and converges to:

$$
\left[\begin{array}{c}
\boldsymbol{\epsilon}_{a v} \\
\boldsymbol{\xi}_{a v}
\end{array}\right] \rightarrow\left[\begin{array}{c}
\left(\tilde{\boldsymbol{\mu}}_{a v}^{T} \boldsymbol{B} \tilde{\boldsymbol{\mu}}_{a v}+\frac{1}{2} \operatorname{Tr}\left(\boldsymbol{A}^{T} \boldsymbol{B} \boldsymbol{A}\right)\right) \\
2 \boldsymbol{B} \tilde{\boldsymbol{\mu}}_{a v}
\end{array}\right]
$$

From (41), we can see that the gradient $\psi$ with respect to $\boldsymbol{\mu}$ is $\nabla_{\boldsymbol{\mu}} \psi(\boldsymbol{\mu})=\boldsymbol{B} \boldsymbol{\mu}$, thus the averaged gradient estimate converges to the gradient of the objective function with respect to the averaged controls.

We now look at the Setpoint Update Step, and present a discrete stability analysis. During the SU step, the 2D-BES controllers move their active power, and reactive power, setpoints based on (26), and afterwards hold their active power, and reactive power, setpoints constant. Thus, we denote the setpoints for the $b^{\text {th }} \mathrm{SP}$ and GE as $\hat{\boldsymbol{\mu}}_{b}$. The SU step takes place after the SP and GE steps, with the update law:

$$
\hat{\boldsymbol{\mu}}_{b+1}=\hat{\boldsymbol{\mu}}_{b}+\boldsymbol{K} \boldsymbol{\xi}_{a v}=\hat{\boldsymbol{\mu}}_{b}-2 \boldsymbol{K} \boldsymbol{B} \tilde{\boldsymbol{\mu}}_{b}
$$

Subtracting $\boldsymbol{\mu}^{*}$ from both sides, we can change the coordinates of (51), obtaining:

$$
\tilde{\boldsymbol{\mu}}_{b+1}=\tilde{\boldsymbol{\mu}}_{b}-\boldsymbol{K} \boldsymbol{\xi}_{a v}=\tilde{\boldsymbol{\mu}}_{b}-2 \boldsymbol{K} \boldsymbol{B} \tilde{\boldsymbol{\mu}}_{b} .
$$

To investigate the stability of the update law, we define a Lyapunov Function:

$$
V_{b}\left(\tilde{\boldsymbol{\mu}}_{b}\right)=\tilde{\boldsymbol{\mu}}_{b}^{T} \boldsymbol{G} \tilde{\boldsymbol{\mu}}_{b},
$$

where $\boldsymbol{G}=\boldsymbol{G}^{T} \succ 0$ is a symmetric, positive definite matrix. For $\tilde{\boldsymbol{\mu}}_{b}$ to converge to zero, $V_{b+1} \leq V_{b}$ must also hold, and therefore:

$$
\begin{aligned}
& \tilde{\boldsymbol{\mu}}_{b}^{T} \boldsymbol{G} \tilde{\boldsymbol{\mu}}_{b} \geq \tilde{\boldsymbol{\mu}}_{b+1}^{T} \boldsymbol{G} \tilde{\boldsymbol{\mu}}_{b+1}, \\
& \tilde{\boldsymbol{\mu}}_{b}^{T} \boldsymbol{G} \tilde{\boldsymbol{\mu}}_{b} \geq\left(\tilde{\boldsymbol{\mu}}_{b}-2 \boldsymbol{K} \boldsymbol{B} \tilde{\boldsymbol{\mu}}_{b}\right)^{T} \boldsymbol{G}\left(\tilde{\boldsymbol{\mu}}_{b}-2 \boldsymbol{K} \boldsymbol{B} \tilde{\boldsymbol{\mu}}_{b}\right),
\end{aligned}
$$

which can be rewritten as:

$$
\tilde{\boldsymbol{\mu}}_{b}^{T}\left(-4 \boldsymbol{G} \boldsymbol{K} \boldsymbol{B}+4 \boldsymbol{B}^{T} \boldsymbol{K}^{T} \boldsymbol{G} \boldsymbol{K} \boldsymbol{B}\right) \tilde{\boldsymbol{\mu}}_{b} \leq 0 .
$$

Thus, we can develop a criteria for the gain matrix $\boldsymbol{K}$, as follows:

$$
\begin{aligned}
& -4 \boldsymbol{G} \boldsymbol{K} \boldsymbol{B}+4 \boldsymbol{B}^{T} \boldsymbol{K}^{T} \boldsymbol{G} \boldsymbol{K} \boldsymbol{B} \preceq 0 \\
& \left(-\boldsymbol{I}+\boldsymbol{B}^{T} \boldsymbol{K}^{T}\right) \boldsymbol{G} \boldsymbol{K} \boldsymbol{B} \preceq 0,
\end{aligned}
$$

which is satisfied for $\boldsymbol{K} \preceq \boldsymbol{B}^{-1}$. 